\title{
The Effect of Digital Noise Reduction on Annoyance and Speech Perception in Low and High Acceptable Noise Level Groups
}

\author{
Hemanth Narayan Shetty ${ }^{1 \oplus}$ Navya Bilijagalemole Nanjundaswamy ${ }^{1}$ \\ ${ }^{1}$ Department of Audiology, All India Institute of Speech and Hearing \\ Mysuru, Mysore, Karnataka India \\ Address for correspondence Hemanth Shetty, PhD, Department of \\ audiology, All India Institute of Speech and Hearing Mysuru, \\ Manasagongothri, Mysore, Karnataka, 570006, India \\ Int Arch Otorhinolaryngol 2019;23:e433-e439. \\ (e-mail: hemanthn.shetty@gmail.com).
}

\begin{abstract}
Introduction Studies have reported that although speech perception in noise was unaltered with and without digital noise reduction (DNR), the annoyance toward noise measured by acceptable noise level (ANL) was significantly improved by DNR with the range between 2.5 and $4.5 \mathrm{~dB}$. It is unclear whether a similar improvement would be observed in those individuals who have an $A N L \geq 14 \mathrm{~dB}$ (predictive of poor hearing aid user) often rejects their aid because of annoyance toward noise.

Objectives (a) To determine the effect of activation of DNR on the improvement in the aided ANL from low- and high-ANL groups; and (b) to predict the change in ANL when DNR was activated.

Method Ten bilateral mild to severe sloping sensorineural hearing loss (SNHL) participants in each of the low- and high-ANL groups were involved. These participants were bilaterally fitted with receiver in canal (RIC) hearing aids (Oticon, Smorum, Egedal, Denmark) with a DNR processor. Both SNR-50\% (Signal to noise ratio (in dB) required to achieve $50 \%$ speech recognition) and ANL were assessed in DNR-on and DNR-off listening conditions.

Results Digital noise reduction has no effect on SNR-50 in each group. The annoyance level was significantly reduced in the DNR-on than DNR-off condition in the low-ANL group. In the high-ANL group, a strong negative correlation was observed between the

Keywords

- noise reduction

- hearing aid

- annoyance

- speech perception ANL in DNR off and a change in ANL after DNR was employed in the hearing aid (benefit). The benefit of DNR on annoyance can be effectively predicted by baselineaided ANL by linear regression.

Conclusion Digital noise reduction reduced the annoyance level in the high-ANL group, and the amount of improvement was related to the baseline-aided ANL value.
\end{abstract}

\section{Introduction}

Individuals with cochlear hearing loss frequently complain of difficulties in following speech in noisy conditions. ${ }^{1}$ Kochkin $^{2}$ reported that $\sim 62.3 \%$ of these individuals are dissatisfied with hearing aids, out of which $25.3 \%$ reject their hearing aid because of background noise. To overcome this problem, hearing aid manufacturers have implemented noise reduction algorithms in the hearing aid circuitry. ${ }^{3}$ The noise reduction algorithm analyses the speech (higher modulation depth) from noise (low modulation depth) through identification of the inherent modulation depth. If the speech and noise shares the same frequency band then a gain reduce at the frequency corresponds to noise band. Bentler et $\mathrm{al}^{4}$ reviewed laboratory studies of digital noise reduction (DNR), and her findings showed an equivocal response on the subjective benefit of received

December 21, 2018

accepted

April 1, 2019
DOI https://doi.org/

10.1055/s-0039-1688811. ISSN $1809-9777$.
Copyright $(2019$ by Thieme Revinter

Publicações Ltda, Rio de Janeiro, Brazil
License terms

(ㄷ) (i) $\ominus$ (\$) 
DNR in the real world. However, improvement in speech perception $^{5}$ and a significant reduction in the annoyance level were evident when DNR was active. ${ }^{6-8}$ A reliable clinical measure to document annoyance from a background noise is the acceptable noise level (ANL). ${ }^{9}$ Acceptable noise level is a measure of the willingness to accept background noise while listening to speech. ${ }^{10}$ The ANL is calculated by taking the difference between the most comfortable level (MCL) for running speech and the maximum background noise level (BNL) that a listener is willing to accept. The ANL ranges between -3.5 and $27 \mathrm{~dB} .{ }^{11}$ ANLs could predict a listener's success of hearing aids with $85 \%$ accuracy. ${ }^{12}$ It was found that ANL was significantly improved by $4.2 \mathrm{~dB}$ on average when DNR was activated, ${ }^{13}$ which is circumscribed to the ANL range ( -3 to $14 \mathrm{~dB}$ ) of the participants of the present study. It is pertinent to determine how much reduction in ANL can be observed when DNR is active in low- $(\leq 7 \mathrm{~dB})$ and high-ANL $(\geq$ $13 \mathrm{~dB}$ ) groups, which cannot be inferred from the study of Mueller et al. ${ }^{13}$ This is important because the high-ANL group is the one who often rejects their hearing aid, since they are less able to put up with background noise while listening to speech. ${ }^{14}$ In addition, a clinician can objectively counsel a patient on the amount of noise above which they would be showing annoyance and its improvement after the activation of DNR in the hearing aid. Further, it helps a client to consider in procuring a hearing aid that has the option of DNR, so that rejection can be minimized. Thus, it is relevant to quantify the change in ANL on hearing impaired individual, when DNR is active. The objective of DNR algorithms is to decrease a gain in the frequency regions corresponding to the background noise, which reduces annoyance from background noise and increases the potential of understanding speech in the presence of noise. Thus, it is hypothesized that the effect of DNR may bring out dramatic changes in the annoyance level, especially in individuals who are able to put up with less noise. The following research question is framed: how does the DNR influence the annoyance level and speech perception in the presence of noise in low- and high-ANL groups? The specific objective was designed to determine if the activation of DNR would improve the aided ANL of low- and high-ANL groups. In addition, we sought to predict the change in ANL, if any, when DNR was activated.

\section{Material and Methods}

\section{Participant Inclusion Criteria}

A single blinded simple randomized one-shot postcomparative research design with repeated measures was used to investigate the effect of DNR on annoyance and on speech perception in low- and high-ANL groups. A convenient sampling method was used to select the participants. A total of 20 participants within an age range between $\geq 60$ to $\leq 75$ years old who had acquired bilateral mild to severe sloping sensorineural hearing loss (SNHL) were involved. The participants were grouped into low- $(n=10$; mean $\mathrm{ANL}=5.5$ $\mathrm{dB}$; ANL range: $3-7 \mathrm{~dB})$ and high-ANL $(n=10$; mean ANL $=18.41 \mathrm{~dB}$; ANL range: $3-7 \mathrm{~dB}$ ) based on the scores of the ANL test. The data of the aided ANL and speech perception in the presence of noise were measured from every participant of each group in DNR-activated and DNR-deactivated conditions. The conditions were randomized across the participants. All of the participants had normal middle ear status, as indicated by ' $A$ ' type tympanogram. The participants were native speakers of Kannada and had obtained normal cognitive scores in the mini mental status examination. None of them had previous experience with a hearing aid. Further, the participants had no complaint of neurological, psychological, cognitive, or otological problems. The mean threshold at each frequency from the right and left ears in the lowand high-ANL groups is represented in - Fig. 1. In the present study, all of the testing procedures used noninvasive techniques, and all of the procedures were explained to the participants before testing. Informed consent was obtained from all of the participants. The present study was approved by the ethical committee of the institution.

\section{Hearing Aids}

All of the participants were bilaterally fitted with two receiver in canal (RIC) hearing aids. Each of the hearing aids had eight channels, directionality was disabled, and adaptive feedback cancellation was enabled. The DNR algorithm was activated, which is modulation based, ${ }^{15}$ and its strength was programmed to 'maximum'. It reduces channel specific gain when the dominant signal in the channel is in a relatively steady state. The National Acoustic Laboratories

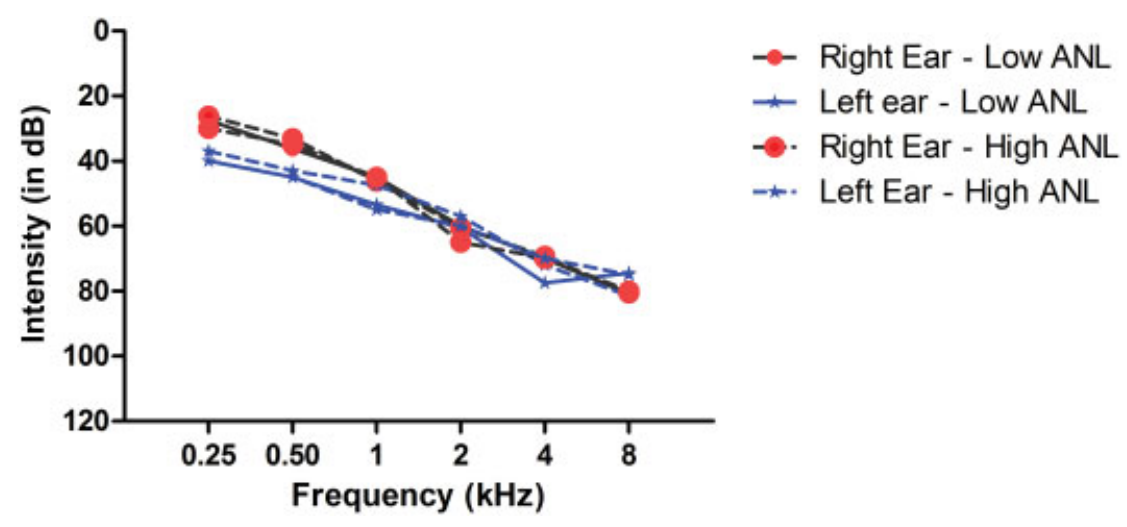

Fig. 1 Mean right and left audiogram of low- and high-ANL groups. Abbreviation: ANL, acceptable noise level. 
nonlinear 1 (NAL NL-1) prescriptive formula was used to program the RIC hearing aids. The compression knee points across channels in the two hearing aids set by the hearing thresholds of the participants were unaltered. All of these parameters were selected in program 1 . These parameters were copied in program 2 with the DNR algorithm deactivated. Real ear insertion gain (REIG) was conducted to verify the hearing aid gain matched to prescriptive target Byrne et al. $^{16}$ It was ensured that the hearing aid gain across frequencies was almost matched to the prescriptive target.

\section{Acceptable Noise Level}

Acceptable noise level is the willingness of the participant to accept background noise while listening to speech. The procedure utilized to assess ANL was the method adopted by Nabelek et al. ${ }^{10}$ The ANL was calculated by subtracting the accepted background noise level from the MCL. The recorded Kannada passage developed and standardized by Sairam et al ${ }^{17}$ was routed through an audiometer (Grason-Stadler, Eden Prairie, Minnesota, USA) at $50 \mathrm{~dB}$ SPL. The output of the audiometer was delivered through a loudspeaker positioned at a distance of 1 meter at a $0^{\circ}$ azimuth. To determine the MCL, the intensity level of the recorded Kannada passage was initially increased in $+5 \mathrm{~dB}$ steps. Further, when the level reached approximately the $\mathrm{MCL}, \mathrm{a}+1 \mathrm{~dB} U \mathrm{Up}$ (no response) -2 $\mathrm{dB}$ (response) step size was utilized to obtain MCL. At the MCL, the speech babble noise was routed through the same loudspeaker, from which the speech passage was delivered. Initially, the noise was presented continuously at $40 \mathrm{~dB}$ SPL, and then the level of noise was gradually increased until the participant was able to put up with the noise with no annoyance and to follow the message. The level at which the individual is able to accept the background noise while listening to speech is the background noise level (BNL). An ANL score between $<1 \mathrm{~dB}$ to $\leq 7 \mathrm{~dB}$ was considered as the low-ANL group. However, those participants who received an ANL score $\geq 13 \mathrm{~dB}$ were considered as the high-ANL group. The measurement of the ANL was repeated twice, and the intraclass correlation found was of 0.98 for the low-ANL group, and of 0.96 for the high-ANL group. In addition, the measurement of the ANL was performed in DNR-on and in DNR-off conditions. The listening conditions were randomized and counterbalanced across the participants of the present study.

\section{Speech Perception in the Presence of Noise}

Two lists of standardized Kannada sentences for recognition developed by Geetha et al $^{18}$ were selected. To assess SNR-50\% in two different experimental conditions, two lists were involved to avoid familiarity with the sentences. Each list comprised 10 sentences, and each sentence had 5 target words. The root mean square (RMS) of each sentence was identified, and then noise was added at the desired signal to noise ratio (SNR). The 10 sentences were mixed with speechshaped noise at different SNRs ranging from $+12 \mathrm{~dB}$ to $6 \mathrm{~dB}$ in $2 \mathrm{~dB}$ step sizes. The noise level remained fixed, and the level of the sentence varied to generate the specified SNRs. Ten sentences embedded at different SNRs were randomized. Each sentence was presented at the MCL of the participants through the loudspeaker, which was located at $0^{\circ}$ azimuth, positioned at a distance of 1 meter from the ear that was being tested of the participant. The participant was instructed to repeat the accurate keywords of each sentence heard. The SNR level at which the testing started $(\mathrm{L})$ and the number of recognized target words in each sentence was noted down. The total number of target words from all of the sentences were added (T). In addition, the total number of words per decrement $(\mathrm{W})$ and the SNR decrement step size in each sentence (d) were noted down. The values obtained were substituted by the given equation adapted by Spearman-Karber to determine SNR $50 \%{ }^{19}$. The equation presented below was used to calculate the SNR 50. The SNR 50 was assessed in DNR-on and in DNR-off conditions. These conditions were counterbalanced and randomized across the participants.

$$
50 \% \text { point }=\mathrm{L}+\left(0.5^{*} \mathrm{~d}\right)-\mathrm{d}(\mathrm{T}) / \mathrm{W}
$$

\section{Statistical Analyses}

The study results were statistically analyzed using the IBM SPSS Statistics for Windows, Version 20.0 (IBM Corp., Armonk, NY, USA). The data of ANL obtained in three listening conditions (unaided, DNR-on, and DNR-off) from each group were subjected to a one-way repeated measure analysis of variance (ANOVA). The Bonferroni post hoc analysis was performed separately for each group to investigate in which condition an annoyance had reduced. In addition, in each group, a dependent samples t-test was performed to assess the difference between DNR-on and DNR-off conditions on SNR 50. Further, the Pearson correlation test was used to investigate the relation between the benefit of DNR and annoyance level to noise in the aided DNR-off condition. A simple linear regression was used to predict the benefit from DNR on annoyance from aided ANL in the high-ANL group.

\section{Results}

The purpose of the present study was to investigate whether the acceptance toward noise reduces when DNR was activated in the hearing aid, in individuals from the low- and high-ANL groups. The ANL was measured in three listening conditions (unaided, DNR-on, and DNR-off). The dependent variable was $A N L$, and the independent variable was the three different listening conditions. The data of ANL obtained in the three listening conditions from the low-ANL group were subjected to a one-way repeated measure ANOVA. The results of the one-way repeated-measures ANOVA showed that there was a significant main effect of reduction of annoyance $(F(1,9)=22.38, p=0.001)$. To see in which listening condition an annoyance had reduced, a Bonferroni post hoc analysis was performed. The results revealed that the annoyance toward noise was significantly more reduced when DNR was on than when DNR was off $(p=0.009)$, as well as than in the unaided condition ( $p=0.001)$ conditions. In addition, annoyance was significantly increased when DNR was off in comparison with the unaided condition $(p=0.001)$. A similar test was performed to determine the 


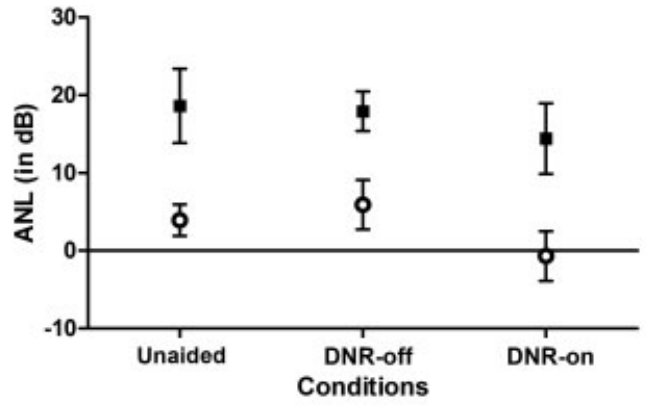

Fig. 2 Acceptable noise level obtained in three listening conditions from both low- and high- ANL groups. Abbreviations: ANL, acceptable noise level; DNR, digital noise reduction

annoyance in the three listening conditions in the high-ANL group. There was a significant main effect of annoyance on the three listening conditions $(\mathrm{F}(1,9)=27.95, p=0.000)$. A post hoc analysis revealed a significant reduction in annoyance when DNR was active than in unaided condition $(p=0.001)$. The annoyance toward noise observed was high when DNR was off than unaided $(p=0.159)$ and DNR on condition $(p=1.012)$, these differences have failed to reach significance. These findings suggest that the annoyance level reduced more when the DNR was active than in the DNR-off condition in both the low- and high-ANL groups (-Fig. 2).

The mean SNR at which the participants of the low- and high-ANL groups had achieved a 50\% speech recognition score in different listening conditions are shown in - Fig. 3. It was observed that, in each group, the SNR 50 was achieved at lesser SNRs in the DNR-on than in the DNR-off condition. A dependent samples t-test revealed no significant effect of SNR between DNR-on and DNR-off conditions [t $(-0.439)$; $p=0.671]$ in the low-ANL group, and [t $(-1.816) ; p=0.103]$ in the high-ANL group, which allows inferring that the implementation of DNR has no effect on the scores of SNR 50 either in individuals who are less annoyed or more annoyed by noise.

\section{SNR $50 \%$}

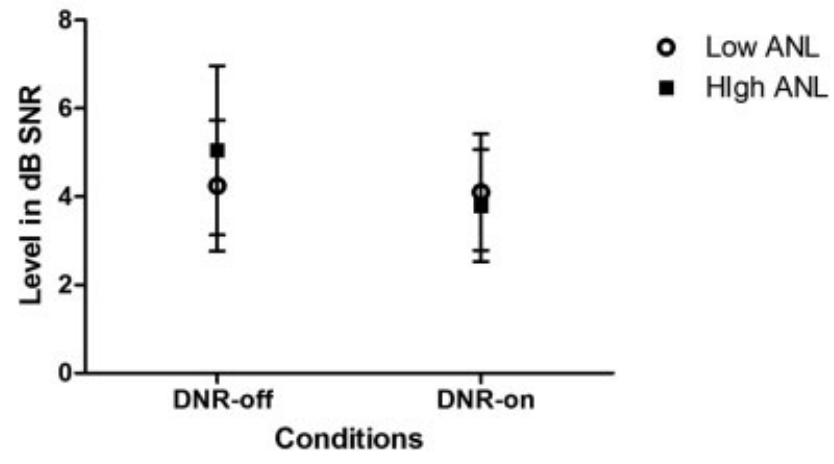

Fig. 3 SNR 50\% (in dB signal-to-noise ratio) in DNR-on and DNR-off conditions obtained from low- and high-ANL groups. Abbreviations: ANL, acceptable noise level; DNR, digital noise reduction

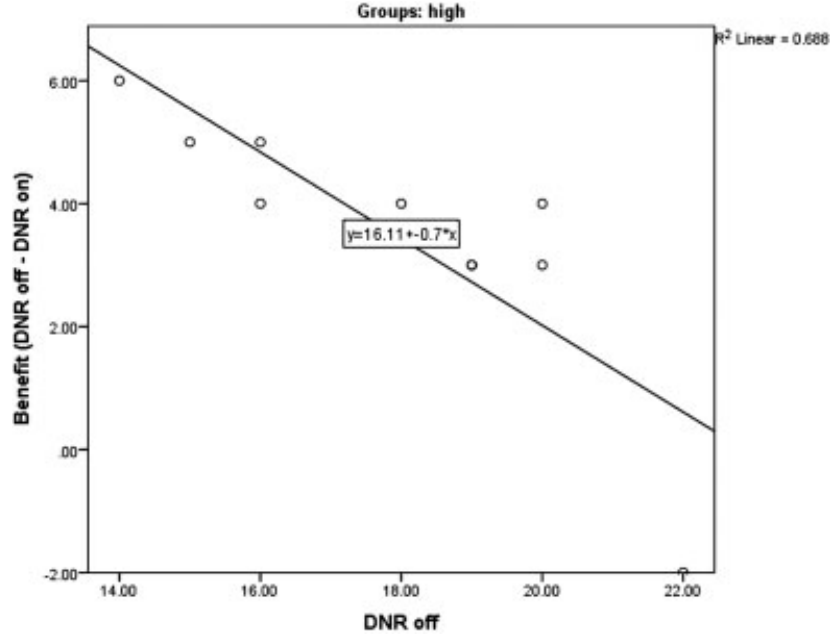

Fig. 4 In the high-ANL group, the $x$-axis indicated the baseline acceptable noise level (ANL) with digital noise reduction (DNR) off, and the $y$-axis shows the change in ANL when DNR was on. The solid line shows the linear regression of the data.

The $2^{\text {nd }}$ purpose of the present study was to investigate the relation between the benefit of DNR and the annoyance level to noise in aided DNR-off condition. A Pearson correlation was found significant, which is strong negatively related $(n=10 ; r=-0.829 ; p=0.003)$ in the high-ANL group. In -Fig. 4, it is observer that, in the high-ANL group, those who had a lower ANL value for the aided DNR-off condition tended to show the most improvement for the DNR-on condition. However, in the low-ANL group, it was observed that there was no relationship between the benefit of DNR over the annoyance value for the aided DNR-off condition $(n=10 ; r=-0.33 ; p=0.034)$.

The $3^{\text {rd }}$ purpose was to predict the benefit from DNR on annoyance from aided ANL in the high-ANL group, which was analyzed using a simple linear regression. The slope coefficient and intercept for benefit from DNR were $-0.785 \mathrm{~dB}$ and $16.12 \mathrm{~dB}$, respectively. The change in ANL was predicted by the formula $[\mathrm{y}=16.12-0.715(\mathrm{x})](\mathrm{y}=$ change in ANL in DNR-on condition; and $\mathrm{x}=$ annoyance level in hearing aid without DNR) when DNR was active. The slope of the regression function is $\sim 0.78 \mathrm{~dB} / \mathrm{dB}$, suggesting that if the aided ANL value is $14 \mathrm{~dB}$, the annoyance toward noise was reduced by $7.8 \mathrm{~dB}$ when DNR was active (a predicted change in annoyance would be $6.31 \mathrm{~dB}$ ). The $\mathrm{R}^{2}$ value was 0.688 , so $68.8 \%$ of the variation in benefit from DNR on annoyance can be explained by the model containing annoyance level only in DNR-on and DNR-off conditions.

\section{Discussion}

It was observed that, although speech perception showed no significant improvement, the majority of listeners (20 out of 22) preferred DNR-on for listening to speech in the presence of noise than the DNR-off condition. The reason for this preference was explained by Mueller et al. ${ }^{13}$ who reported a benefit (reduction) of $4.5 \mathrm{~dB}$ in annoyance when DNR was employed in the hearing aid in the study participants who 
had an ANL range between 6 and $14 \mathrm{~dB}$. The range of ANL in the participants of the Muller's study had within the successful and average hearing aid users 14 in whom $86.3 \%$ of participants had met the criterion value of $10 \mathrm{~dB}$ in ANL when DNR was on. The benefit of $4.5 \mathrm{~dB}$ from activation of DNR on annoyance towards noise cannot be leveled on those participants who have ANL value of $\geq 14 \mathrm{~dB}$ involves subtle difference in physiological mechanism in them. ${ }^{20}$ Thus, it is of utmost importance to determine how much change in annoyance toward noise is observed when DNR is activated, especially in the high-ANL group ( $>13 \mathrm{~dB}$ ). The participants of the high-ANL group are the ones who often reject their hearing aid due to background noise. ${ }^{14}$ Kochkin $^{2}$ reported that $\sim 25.3 \%$ of hearing aid users reject their hearing aid because of background noise. As shown in - Fig. 2, the ANL was significantly more reduced when DNR was active than in the unaided and in the DNR-off condition in the low-ANL group. The level required to achieve MCL was almost the same between DNR-on and DNR-off as the sentences were presented in quiet condition (-Fig. 5A). However, the participants were able to put up with more noise when DNR was employed than in the DNR-off condition (-Fig. 5A), which allows inferring that, in the DNR-on condition, the speechshaped noise has reduced the gain, especially in speech valleys, in interword, and in intersentence pauses, ${ }^{8}$ which enabled them to put up with a higher level of background noise to obtain a small value of ANL. In the high-ANL group, although the annoyance level was reduced in the DNR on than in the DNR-off condition, it failed to reach statistical significance. - Fig. 5B shows that the MCL was almost the same between DNR-on and DNR-off conditions, but the standard deviation (SD) of the BNL was higher in DNR-on than in DNR-off, which has also caused a high SD in the ANL value, which speculates the insensitivity of efferent auditory pathway. This speculation is supported by the research report by Harkrider et $\mathrm{al}^{20}$ who reported a weaker efferent mechanism in the high-ANL group, which reduced the capacity of inhibition. Thus, the participants of the highANL group are unable to put up with more noise. Even in speech understanding in the presence of noise, the partici- pants of the high-ANL group required a higher SNR for 50\% recognition of speech. In - Fig. 4, it is observed that the SNR required to achieve 50\% recognition was higher in the DNRoff condition than in the DNR-on condition, but failed to reach statistical significance for the high-ANL group. However, irrespective of listening condition (DNR-on of DNR-off) the SNR required to achieve 50\% speech recognition was almost the same in the low-ANL group. The findings are in consonance with the findings of Mueller et al, ${ }^{13}$ who reported that DNR has no effect on speech in the presence of noise.

Nabelek ${ }^{14}$ has suggested that an aided ANL of around 10 $\mathrm{dB}$ or better is predictive of successful hearing aid use. Thus, a question arises: did the change in DNR on annoyance reach the above specified criteria of successful hearing aid use? To answer this question, we have plotted individual ANL data for the low- and high-ANL groups for DNR-on and DNR-off conditions (-Fig. 6). The range of ANL observed was between 1 and $9 \mathrm{~dB}$ in the low-ANL group for the DNR-off condition, and its range of ANL changed to between - 5 to $3 \mathrm{~dB}$ when DNR was employed. Exactly $40 \%$ of the participants (4/10) had an aided ANL $>7$ to $9 \mathrm{~dB}$ which is predictive of average hearing aid users whereas with DNR-on, all of the 4 participants had an ANL of $\leq 7 \mathrm{~dB}$ or better. However, in the highANL group, except in 1 participant, an improvement in ANL was observed when DNR was employed in the hearing aid with a range between 7 to $20 \mathrm{~dB}$, but in the DNR-off condition, the range of ANL was between 14 and $27 \mathrm{~dB}$. Specifically, the DNR on condition has changed the annoyance towards noise in 4 out of 10 participants and met the range of $<7$ to $\geq 14 \mathrm{~dB}$ in ANL, which increases their probability of average success with the hearing aid. ${ }^{14}$

We have questioned whether the change in ANL with DNR-on might be related to baseline-aided ANL in both lowand high-ANL groups. In the low-ANL group, the change in ANL in the DNR-on condition was almost the same, irrespective of the ANL value for the DNR-off condition, which reflected no relationship between the ANL value for DNRoff as a function of change in the ANL value for the DNR-on condition. However, in the high-ANL group, a significant
Low ANL group

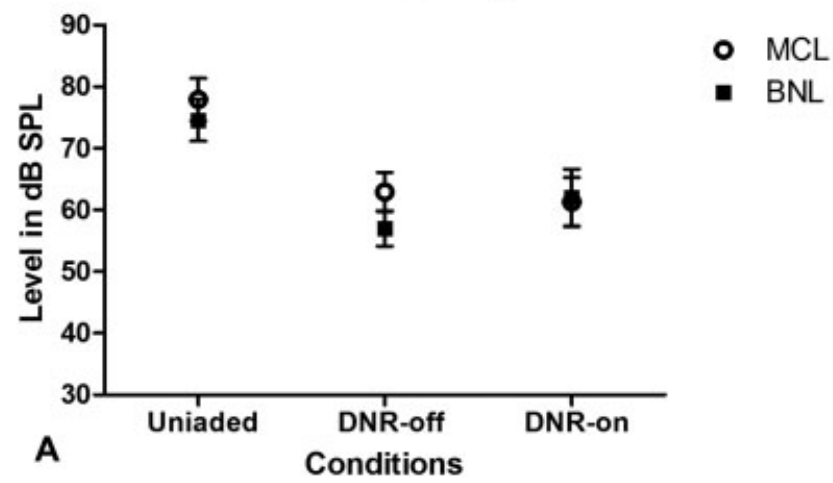

High ANL group

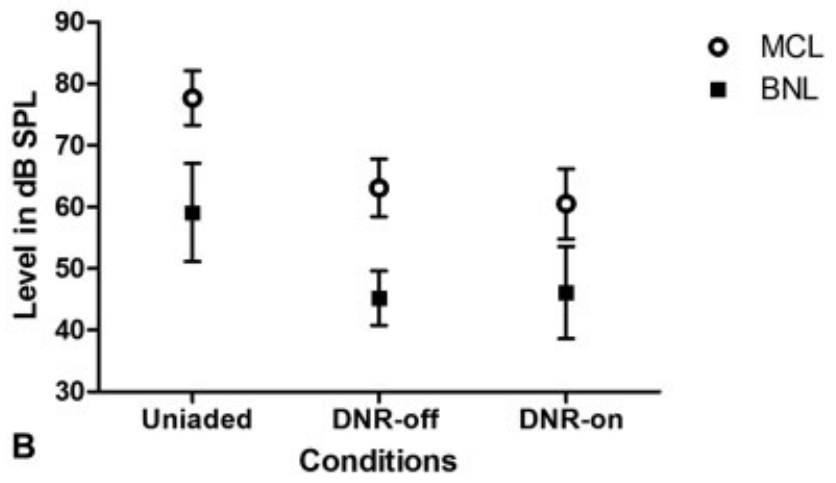

Fig. 5 (a) Low-ANL group (b) high-ANL group. Mean most comfortable levels (MCLs; unfilled circles) for speech and mean acceptable background noise levels (BNLs; filled squares) for speech-shaped noise presented with speech were obtained in each of the listening conditions (unaided, aided-DNR on, and aided-DNR off). Abbreviation: DNR, digital noise reduction. 


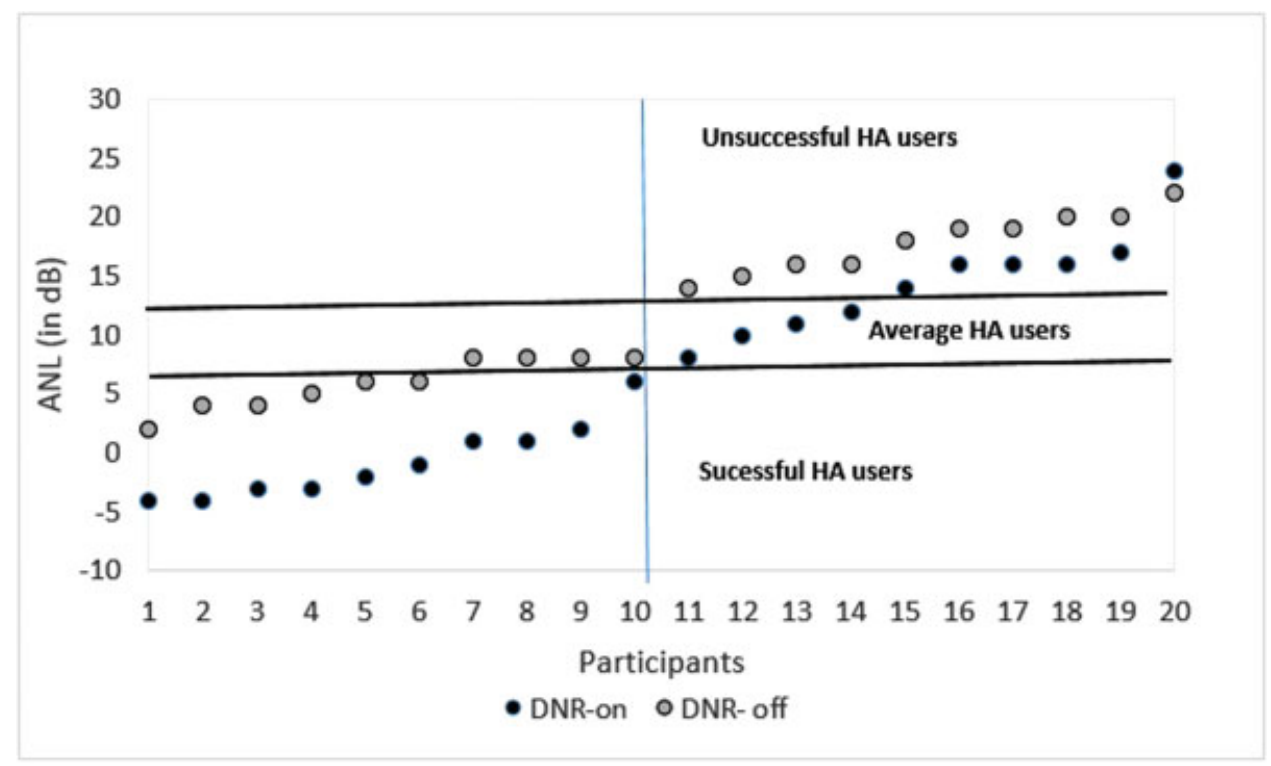

Fig. 6 Acceptable noise level is plotted for DNR-on and -off conditions for each of the 20 participants. The horizontal line represents a predictive criteria of successful hearing aid user. Vertical line separates two groups (low and high) based on baseline unaided ANL.

strong negative correlation was observed between a change in the ANL with DNR-on and with aided DNR-off. In - Fig. $\mathbf{6}$, the data suggests that those who had a smaller ANL for the aided DNR-off condition tended to show the most improvement for the DNR- on condition. The finding of the present study is in consonance with the research conducted by Lowery et $\mathrm{al}^{6}{ }^{6}$ who suggested that DNR reduced ANL, and that the amount of reduction is based on the baseline value. The relationship between ANL in DNR off condition and the change in ANL after DNR was active in hearing aid (benefit) revealed negative correlation in high ANL group. It was observed that those who had a lower ANL value for the aided condition tended to show the most improvement (change/ benefit) in the DNR-on condition. The regression analysis was conducted to predict the benefit of DNR on annoyance from aided ANL. It was observed that the change in annoyance was predicted to be $6.3 \mathrm{~dB}$ when DNR was active in the hearing aid in whom the aided ANL was $14 \mathrm{~dB}$. However, an individual who presented with an aided ANL of $21 \mathrm{~dB}$, a change in ANL predicted after DNR-on would be of $3.4 \mathrm{~dB}$, which allows inferring that, in the high-ANL group, those who have a lower ANL value would obtain a relatively higher benefit than those who have a high ANL value.

\section{Conclusion}

The scores of SNR 50 are unaffected by amplification in each group, but the annoyance has reduced when DNR was employed in each group. In the high-ANL group, the baseline-aided ANL determined the amount of benefit achieved when DNR was activated in the hearing aid.

Research involving Human Participants

The present study was approved by the All India Institute of Speech and Hearing institutional research ethical com- mittee and was performed in accordance with the ethical standard as laid down by the review board.

\section{Informed Consent}

Informed consent was obtained from all of the individual participants included in the present study.

\section{Ethical Approval}

All of the procedures performed in studies involving human participants were in accordance with the ethical standards of the All India Institute of Speech and Hearing institutional research committee and its later amendments or comparable ethical standards.

Funding Source

None.

Conflicts of Interests

The authors have no conflicts of interests to declare.

\section{References}

1 Cord MT, Surr RK, Walden BE, Dyrlund O. Relationship between laboratory measures of directional advantage and everyday success with directional microphone hearing aids. J Am Acad Audiol 2004;15(05):353-364

2 Kochkin S. Consumers rate improvements sought in hearing instruments. Hear Rev 2002;9(11):18-22

3 Schum DJ. Noise-reduction circuitry in hearing aids:(2) Goals and current strategies. Hear J 2003;56(06):32-33

4 Bentler R, Chiou LK. Digital noise reduction: an overview. Trends Amplif 2006;10(02):67-82. Epub 2006/09/09. doi: 10.1177/ 1084713806289514. PubMed PMID: 16959731; PubMed Central PMCID: PMCPMC4111515.

5 Walden BE, Surr RK, Cord MT, Edwards B, Olson L. Comparison of benefits provided by different hearing aid technologies. J Am Acad Audiol 2000;11(10):540-560 
6 Lowery KJ, Plyler PN. The effects of noise reduction technologies on the acceptance of background noise. J Am Acad Audiol 2013;24 (08):649-659

7 Ricketts TA, Hornsby BW. Sound quality measures for speech in noise through a commercial hearing aid implementing digital noise reduction. J Am Acad Audiol 2005;16(05):270-277

$8 \mathrm{Wu}$ Y-H, Stangl E. The effect of hearing aid signal-processing schemes on acceptable noise levels: perception and prediction. Ear Hear 2013;34(03):333-341

9 Nabelek AK, Tampas JW, Burchfield SB. Comparison of speech perception in background noise with acceptance of background noise in aided and unaided conditions. J Speech Lang Hear Res 2004;47(05):1001-1011

10 Nabelek AK, Tucker FM, Letowski TR. Toleration of background noises: relationship with patterns of hearing aid use by elderly persons. J Speech Hear Res 1991;34(03):679-685

11 Plyler PN, Alworth LN, Rossini TP, Mapes KE. Effects of speech signal content and speaker gender on acceptance of noise in listeners with normal hearing. Int J Audiol 2011;50(04): 243-248. Doi: 10.3109/14992027.2010.545082

12 Freyaldenhoven MC, Plyler PN, Thelin JW, Muenchen RA. Acceptance of noise growth patterns in hearing aid users. J Speech Lang Hear Res 2008;51(01):126-135. Doi: 10.1044/1092-4388(2008/ 009)
13 Mueller HG, Weber J, Hornsby BW. The effects of digital noise reduction on the acceptance of background noise. Trends Amplif 2006;10(02):83-93. Epub 2006/09/09. doi: 10.1177/ 1084713806289553. PubMed PMID: 16959732; PubMed Central PMCID: PMCPmc4111517

14 Nabelek AK, Freyaldenhoven MC, Tampas JW, Burchfiel SB, Muenchen RA. Acceptable noise level as a predictor of hearing aid use. J Am Acad Audiol 2006;17(09):626-639

15 Powers T, Holube I, Wesselkamp M. The use of digital features to combat background noise. High Performance Hearing Solutions. 1999;3:36-39

16 Byrne D, Dillon H, Ching T, Katsch R, Keidser G. NAL-NL1 procedure for fitting nonlinear hearing aids: characteristics and comparisons with other procedures. J Am Acad Audiol 2001;12(01):37-51

17 Sairam V, Manjula P. Long term average speech spectrum in Kannada. University of Mysore; 2002

18 Geetha C, Kumar K, Manjula P, Pavan M. Development and standardisation of the sentence identification test in the Kannada language. J Hear Sci 2014;4(01):18-26

19 Finney DJ. Statistical method in biological assay: Charles Griffin: London; 1952

20 Harkrider AW, Smith SB. Acceptable noise level, phoneme recognition in noise, and measures of auditory efferent activity. J Am Acad Audiol 2005;16(08):530-545 